\title{
Análisis del uso de las redes sociales de las instituciones de educación superior catalanas
}

\author{
Marc Pérez-Bonaventura ${ }^{1}$ (D), Salomé Tárrega² ${ }^{\text {(D) }}$, Jordi Vilajosana ${ }^{3}$
}

Artículo de investigación. Recibido: 20/05/2020. Aceptado: 02/09/2020. Publicación avanzada: 04/11/2020. Publicado: 04/01/2021.

\begin{abstract}
Resumen
INTRODUCCIÓN. En la actualidad, la competencia entre las universidades ha aumentado de forma importante y los alumnos pueden elegir entre muchas opciones. En este contexto, las universidades necesitan el marketing para destacar y ser las elegidas. En la era de Internet $y$ la web 2.0 parte de la comunicación universitaria se ha trasladado a las redes sociales y estas son una de las mejores formas para llegar a los estudiantes. Este trabajo investiga las redes sociales de las universidades de Cataluña.
\end{abstract}

MÉTODO. Se han estudiado los seguidores, las publicaciones y la interacción que consiguen en Facebook, Instagram, Twitter y YouTube las doce universidades catalanas. Se recopilaron los datos en dos momentos: entre el 15 y el 21 de febrero de 2018 (seguidores) y entre el 20 y el 26 de junio de 2018 (todas las variables).

RESULTADOS. Se aprecia un uso muy desigual de las redes entre las diferentes universidades y también en una misma universidad. Se detectan diferencias importantes entre los resultados en valores absolutos y relativos, así como entre las universidades públicas y privadas.

DISCUSIÓN. Se observa una falta de planificación y estrategia en las redes sociales por parte de las universidades, acentuada en las universidades públicas en contraste con las privadas.

\section{Palabras clave}

Educación; Marketing digital; Redes sociales; Gestión de universidades; Educación superior; Uso de las redes sociales.

\section{Datos de los autores}

1 Universitat Politècnica de Catalunya, España. Departamento de Organización de Empresas. Correo para la correspondencia: marcperezbonaventura@gmail.com

${ }^{2}$ Universitat Oberta de Catalunya (UOC); Universitat de Vic-Universitat Central de Catalunya (UVic-UCC), España. Estudios de Psicología y Ciencias de la Educación (UOC); Facultad de Ciencias de la Salud de Manresa (UVic-UCC).

${ }^{3}$ Universitat Politècnica de Catalunya, España. Departamento de Organización de Empresas.

\section{Referencia recomendada}

Pérez-Bonaventura, M., Tárrega, S., y Vilajosana, J. (2021). Análisis del uso de las redes sociales de las instituciones de educación superior catalanas. REIRE Revista d'Innovació i Recerca en Educació, 14(1), 1-20. https://doi.org/10.1344/reire2021.14.131558

(C) 2021 Los autores. Este artículo es de acceso abierto sujeto a la licencia Reconocimiento 4.0 Internacional de Creative Commons, la cual permite utilizar, distribuir y reproducir por cualquier medio sin restricciones siempre que se cite adecuadamente la obra original. Para ver una copia de esta licencia, visite https://creativecommons.org/licenses/by/4.0/ 


\section{Títol (català)}

Anàlisi de l'ús de les xarxes socials de les institucions d'educació superior catalanes

\section{Resum}

INTRODUCCIÓ. En l'actualitat, la competència entre les universitats ha augmentat de forma important i els alumnes poden triar entre moltes opcions. En aquest context, les universitats necessiten el màrqueting per destacar i per ser les escollides. En l'era d'Internet $\mathrm{i}$ el web 2.0 part de la comunicació universitària s'ha traslladat a les xarxes socials, i aquestes són una de les millors maneres d'arribar als estudiants. Aquest treball investiga les xarxes socials de les universitats de Catalunya.

MÈTODE. S'han estudiat els seguidors, les publicacions i la interacció que aconsegueixen a Facebook, Instagram, Twitter i YouTube les dotze universitats catalanes. Es van recopilar les dades en dos moments: entre el 15 i el 21 de febrer de 2018 (seguidors) i entre el 20 i el 26 de juny de 2018 (totes les variables).

RESULTATS. S'aprecia un ús molt desigual de les xarxes entre les diferents universitats i també en una mateixa universitat. Es detecten diferències importants entre els resultats en valors absoluts i relatius, així com entre les universitats públiques i privades.

DISCUSSIÓ. S'observa una manca de planificació i estratègia a les xarxes socials per part de les universitats, accentuada a les universitats públiques en contrast amb les privades.

Paraules clau

Educació; Màrqueting digital; Xarxes socials; Gestió d’universitats; Educació superior; Ús de les xarxes socials.

\section{Title (English)}

An analysis of the use of social networks in Catalan higher education institutions

\section{Abstract}

INTRODUCTION. Competition between universities has increased significantly in recent times and students can now choose from many options. In this new context, universities need to use marketing in order to stand out from their competitors. In the age of Internet and Web 2.0, universities have transferred part of their communication to the social networks, an environment that is an ideal vehicle for reaching students. The present study investigates Catalan universities social networks.

METHOD. The study focused on 12 Catalan universities and their followers, publications and interactions on Facebook, Instagram, Twitter and YouTube. Data were collected at two time points; between 15 and 21 February 2018 (followers) and between 20 and 26 June 2018 (all variables).

RESULTS. The use of social networks varied widely both between different universities and inside the same university. Significant differences were detected between the results in both absolute and relative values, and also between public and private universities.

DISCUSSION. A lack of planning and strategy regarding social networks was observed among the universities. This lack of planning was more notable among the publicly funded universities than in the private ones.

\section{Keywords}

Education; Digital marketing; Social networks; University management; Higher education; Use of social networks. 


\section{Introducción}

Hace años que ha aumentado de forma considerable la competencia entre las universidades a nivel global (Varghese, 2008), por lo que ante este entorno competitivo el marketing ha ganado importancia para las universidades (Trullàs, 2015), así como también la comunicación. Ambos han sido considerados herramientas necesarias para ayudar a destacar y tratar de influir en el proceso de elección de universidad de los futuros estudiantes (Carmelo García, 2007) y para asegurar su supervivencia a largo plazo, tanto de universidades públicas como privadas (Trullàs, 2015). En este sentido, algunos autores han señalado que los gabinetes de comunicación de las universidades son esenciales para mantener una relación fluida entre universidad y estudiantes de nuevo acceso o ya matriculados en cursos anteriores (García Orosa, 2009), siendo Internet el medio más frecuente de comunicación por parte de las universidades (Carmelo García, 2007). Hace años ya se consideró importante que las universidades incluyeran estrategias de marketing digital y herramientas de comunicación 2.0 como las redes sociales (San Millán Fernández et al., 2008), y que los gabinetes de comunicación de las universidades adquirieran la responsabilidad de gestionar la actividad de las universidades en Internet y redes sociales, además del trabajo de comunicación organizacional tradicional (García Orosa, 2009).

En la última década, Internet es el medio más utilizado para la comunicación universitaria y el marketing superando a otros medios como prensa, radio o televisión (Wang, 2011). En concreto, las redes sociales (RRSS) parecen haberse ido imponiendo como una poderosa herramienta de marketing digital e influencia en la elección de la universidad (Wankel y Wankel, 2011), considerándose la punta de lanza en la comunicación de las instituciones de educación superior en Internet por algunos autores (Kimmons et al., 2017).

Ante tales consideraciones, varios autores han señalado la relevancia de una buena planificación, y de un diseño adecuado de la estrategia de actuación en el uso de las RRSS. Por ejemplo, Brito et al. (2012) recomiendan la planificación de acciones concretas, tratar de estar presentes en varias RRSS creando sinergias entre ellas, crear grupos de interés específicos, crear contenido que sea de interés para los usuarios que potencie la interacción y tener un equipo específico para llevar las RRSS de forma profesional. En la misma línea, Reina Estévez et al. (2012) recomiendan cumplir una regularidad en la frecuencia de las publicaciones y sus horarios, planificar los contenidos de las publicaciones y responder a los comentarios de los usuarios cuando los realizan.

A la hora de identificar estrategias y acciones concretas, el estudio de la presencia y de la actividad de los perfiles de las universidades en las RRSS puede proporcionar orientaciones interesantes. Así, un estudio reciente indica que la sola presencia de las universidades en todas las RRSS no es relevante porque lo que incrementa la visibilidad de la marca en RRSS es que las universidades tengan actividad, es decir que creen contenido mediante publicaciones en estas redes (Amaral y Santos, 2020). Por su parte, Segura-Mariño et al. (2020) indican que las RRSS que más utilizan las mejores universidades del mundo son Facebook y Twitter. Además, éstas no focalizan el uso de RRSS para la promoción de la oferta académica, sino que en Facebook publican sobre temas institucionales usando muchos "hashtags", y en Twitter publican para motivar o incentivar la interacción de los usuarios mediante contenidos de investigación o interés social. Para generar estos espacios de comunicación la red social de Twitter sería la más efectiva, pues genera más conversaciones que Facebook. No obstante, Facebook es la red social que más utilizan las universidades para sus publicaciones (Bélanger et al., 2014). 
El público objetivo del marketing de las universidades son los futuros estudiantes, los estudiantes actuales, los exalumnos, las empresas que dan trabajo a los graduados y los que apoyan la universidad a nivel financiero según sea pública o privada (Hayes, 1993), también hay otros públicos minoritarios como los docentes o el personal administrativo. No obstante, el público que más importa a las oficinas de admisión son los futuros estudiantes (Lewison y Hawes, 2007), pues deben elegir la universidad dónde realizar sus estudios de educación superior y preinscribirse. En este sentido, Egan (2011) señala el gran peso que tienen actualmente las RRSS cuando los consumidores necesitan tomar una decisión. En la toma de decisión de su futura universidad, los alumnos adoptan el comportamiento de un consumidor, así evalúan las alternativas y eligen el servicio que creen que satisface mejor sus necesidades (Schiffman y Kanuk, 1991). Por este motivo, las universidades se han reenfocado más al mercado y al marketing tratando a sus estudiantes como consumidores (Mazzarol y Soutar, 2002).

Los alumnos que provienen de la enseñanza secundaria, un 96,55 \% de los futuros alumnos universitarios en Cataluña (Generalitat de Catalunya, 2017), a final de curso deben decidir la universidad para cursar sus futuros estudios universitarios. La preinscripción universitaria tiene como plazo de la convocatoria del 4 de junio al 1 de julio (Generalitat de Catalunya, 2019). En este momento las universidades hacen esfuerzos comunicativos para atraer a los nuevos estudiantes y ser elegidas por lo que es un momento clave para ellas.

En consonancia con la relevancia de las RRSS como medio de comunicación argumentado anteriormente, es importante conocer las tendencias digitales de estos alumnos. Benedicto et al. (2017) explican que el 88 \% de los jóvenes de España utilizan Internet diariamente, y estudios más recientes elevan los usuarios frecuentes de entre dieciséis y veinticuatro años al 93,6 \% (Fundación Telefónica, 2020). Los motivos prioritarios de conexión a Internet de estos son el visionado de vídeos y la participación en RRSS (Fundación Telefónica, 2016).

En cuanto al uso de estas RRSS, algunos autores señalaban que las universidades españolas habían tenido dificultades para adaptarse al entorno 2.0 tanto a nivel de aprendizaje como a nivel del uso de marketing digital (Freire, 2008) y que las universidades españolas utilizaban Internet más cómo un canal de información que de comunicación (García Orosa, 2009). Así, tienden a utilizar las RRSS para difundir notas de prensa y eventos, pero su uso para crear contenido es escaso, por ello la interacción con los seguidores es muy escasa (Paniagua Rojano et al., 2012).

Actualmente las RRSS más utilizadas en España son Facebook, WhatsApp, YouTube, Instagram y Twitter siguiendo ese orden (IAB Spain Research, 2018). En cuanto a las universidades españolas, en 2016 la penetración del uso de Facebook, Twitter y LinkedIn es del 93,9 \% y de YouTube del 89,1 \%. Es decir, existe una presencia importante de las universidades en las RRSS. Sin embargo, se critica una falta de estrategia en la actuación de las universidades españolas en las RRSS (Zarco et al., 2016). Asimismo, parece que las instituciones de educación superior no tienen un sistema de comunicación homogéneo en RRSS y aplican diferentes políticas comunicativas (Simón Onieva, 2014). Otros autores señalan una falta de estrategia de marketing digital, sin regularidad ni horarios de publicación preestablecidos, por lo que la forma de trabajar las RRSS por parte de las universidades españolas es inadecuada (Reina Estévez et al., 2012).

Las instituciones de educación superior españolas, a pesar de estar presentes en las RRSS, todavía no acaban de estar bien adaptadas al entorno digital y los autores ponen de manifiesto que no parecen tener estrategias bien definidas de marketing online (Paniagua Rojano et al., 2012). Las universidades usan las RRSS para promocionarse y mejorar su imagen en lugar de para conectar con los usuarios y generar 
interacción (Simón Onieva, 2015). Recientemente, Blázquez et al. (2020) critican la frecuencia de publicación de las universidades españolas y el hecho de que no publican en el momento más adecuado. Asimismo, siguiendo la senda de los otros autores señalan la necesidad de mejorar el desarrollo de las estrategias de marketing que permitan crear espacios de comunicación entre las universidades y los estudiantes y entre los mismos estudiantes.

Actualmente faltan estudios con datos concretos de los perfiles en RRSS de las universidades españolas, y concretamente de las universidades de Cataluña, que permitan valorar su actividad durante estos periodos que podrían ser clave en las RRSS. Este trabajo pretende aportar información sobre la presencia y actividad de los perfiles de las universidades catalanas en las RRSS.

\section{Objetivos}

El presente trabajo pretende analizar el uso y la actividad de las universidades de Cataluña en las RRSS de Facebook, Instagram, Twitter y YouTube y la interacción que obtienen por parte de los usuarios durante el periodo de preinscripción universitaria. Para ello se estudia la frecuencia de las publicaciones que hacen las universidades en las RRSS, el número de seguidores que tienen y la respuesta de los usuarios a las publicaciones de las universidades a partir del número de "Me gusta" durante 7 días en junio de 2018. También se analiza el crecimiento del número de seguidores de febrero a junio de 2018.

\section{Metodología}

Se llevó a cabo un estudio cuantitativo de observación documental, con un diseño prospectivo y descriptivo para todas las variables y, además, un estudio longitudinal para la variable del número de seguidores.

\subsection{Población y muestra}

Según los datos del informe 2015/16 del Ministerio de Educación Cultura y Deporte y la Secretaría General de Universidades (2016) y los datos recientes de la Secretaria d'Universitats i Recerca (2019), actualmente en Cataluña existen 12 universidades. Se han estudiado todas estas analizando privadas y públicas para tratarlas en igualdad de condiciones, pues compiten en un mismo mercado, y poder así establecer comparaciones útiles entre sus actividades y resultados siguiendo la estela de otros estudios (Paniagua Rojano et al., 2012; Zarco et al., 2016). Las universidades estudiadas se detallan en la Tabla 1 señalando si tienen o no actividad en cada una de las RRSS de Facebook, Twitter, Instagram o YouTube. Se han analizado sus cuentas oficiales en las RRSS y se han obviado las cuentas de otros colectivos concretos relacionados con la universidad como sus campus o facultades, para homogeneizar y simplificar la comparación entre las diferentes universidades en su uso de las redes, emulando otros estudios en este campo (Onieva, 2015; Rodríguez Ruibal y Santamaría Cristino, 2012). 
Tabla 1

Universidades catalanas y sus perfiles en RRSS en el momento del estudio

\begin{tabular}{|c|c|c|c|c|c|c|}
\hline Universidades & Acrónimo & Púb./Priv. & Facebook & Twitter & Instagram & YouTube \\
\hline U. Abat Oliba CEU & (UAO-CEU) & Privada & Sí & Sí & Sí & Sí \\
\hline U. Autònoma de Barcelona & (UAB) & Pública & Sí & Sí & Sí & Sí \\
\hline U. de Barcelona & (UB) & Pública & Sí & Sí & Sí & Sí \\
\hline U. de Girona & (UdG) & Pública & Sí & Sí & Sí & Sí \\
\hline U. Internacional de Catalunya & (UIC) & Privada & Sí & Sí & Sí & Sí \\
\hline U. de Lleida & (UdL) & Pública & Sí & Sí & Sí & Sí \\
\hline U. Oberta de Catalunya & (UOC) & Privada & Sí & Sí & Sí & Sí \\
\hline U. Politècnica de Catalunya & (UPC) & Pública & Sí & Sí & Sí & Sí \\
\hline U. Pompeu Fabra & (UPF) & Pública & Sí & Sí & Sí & Sí \\
\hline U. Ramón Llull & (URL) & Privada & Sí & Sí & No & Sí \\
\hline U. Rovira i Virgili & (URV) & Pública & Sí & Sí & Sí & Sí \\
\hline U. de Vic-U. Central de Catalunya & (UVic-UCC) & Privada & Sí & Sí & Sí & Sí \\
\hline
\end{tabular}

Fuente: Elaboración propia.

En la Tabla 1, que muestra la presencia de las universidades en RRSS, se destaca que la URL no tenía presencia en la red social de Instagram durante el periodo de estudio. El resto de las universidades tenían presencia en las cuatro RRSS, aunque a veces no tuvieran actividad en ellas.

\subsection{Variables analizadas}

El número de seguidores en RRSS de una marca o entidad es el número de personas que siguen sus noticias e integran la comunidad de esta marca o entidad en una red social. Los "Me gusta" de los usuarios a las publicaciones de una marca o entidad son una forma de reacción de los usuarios mostrando su interés respecto a sus publicaciones. Las publicaciones son un mensaje, información o estado en formato de texto, foto, vídeo o hipervínculo que las marcas o entidades comparten en su muro de la red social y que los usuarios de la red pueden ver e interactuar con ellas (Rissoan, 2006). En el artículo, como indicador del nivel de actividad de las universidades se ha registrado el número de publicaciones que estas realizaban en las RRSS. Como indicadores de la interacción de los usuarios a la actividad de las universidades se ha registrado el número de seguidores en RRSS, que indica el reconocimiento de la marca o entidad en la red social, y el número de "Me gusta" de los usuarios a sus publicaciones (Ashley y Tuten, 2015; De Vries et al., 2012).

\subsection{Procedimiento}

La recopilación principal de datos se llevó a cabo del día 20 al 26 de junio de 2018, periodo comprendido dentro del final del de preinscripción universitaria -del 4 de junio al 1 de julio de 2018. Se hizo un seguimiento diario de las variables analizadas para cada una de las universidades en las diferentes RRSS durante los siete días del estudio. Además, para analizar la variación del número de seguidores de las universidades en las RRSS se recopilaron los datos en dos momentos: en la semana del 15-21 de febrero de 2018 y en la semana del 20-26 de junio de 2018.

\subsection{Análisis de datos}

El número de seguidores se obtuvo a partir de una media de los datos diarios recopilados durante el periodo considerado. El número de publicaciones y número de "Me gusta" se obtuvo a partir de la suma de los datos diarios reunidos durante el periodo descrito. En el estudio se han analizado valores absolutos y relativos para evitar la distorsión que puede causar el tamaño de las diferentes 
universidades a los números de seguidores y de reacciones. Los valores relativos de los seguidores se han calculado por estudiante, y en el caso de las reacciones se han calculado por cada 1.000 estudiantes. El número de publicaciones se muestra únicamente en valores absolutos porque no se ve afectado por la distorsión. El valor relativo se pondera por el número de estudiantes porque es el público principal de las universidades y sus RRSS. El número de estudiantes de las universidades del curso examinado se ha conseguido a través de las estadísticas del Ministerio de Ciencia Innovación y Universidades (2019). Para la gestión, tratamiento y análisis de los datos se han utilizado los programas Microsoft Access, Microsoft Excel y SPSS.

\section{Resultados}

\subsection{Seguidores en las RRSS}

\subsubsection{Seguidores en las RRSS en valor absoluto}

El número de seguidores de los perfiles de Facebook, Twitter, Instagram y YouTube de cada universidad se muestra en el siguiente gráfico (Figura 1).

\section{Figura 1}

Seguidores de cada universidad en las RRSS

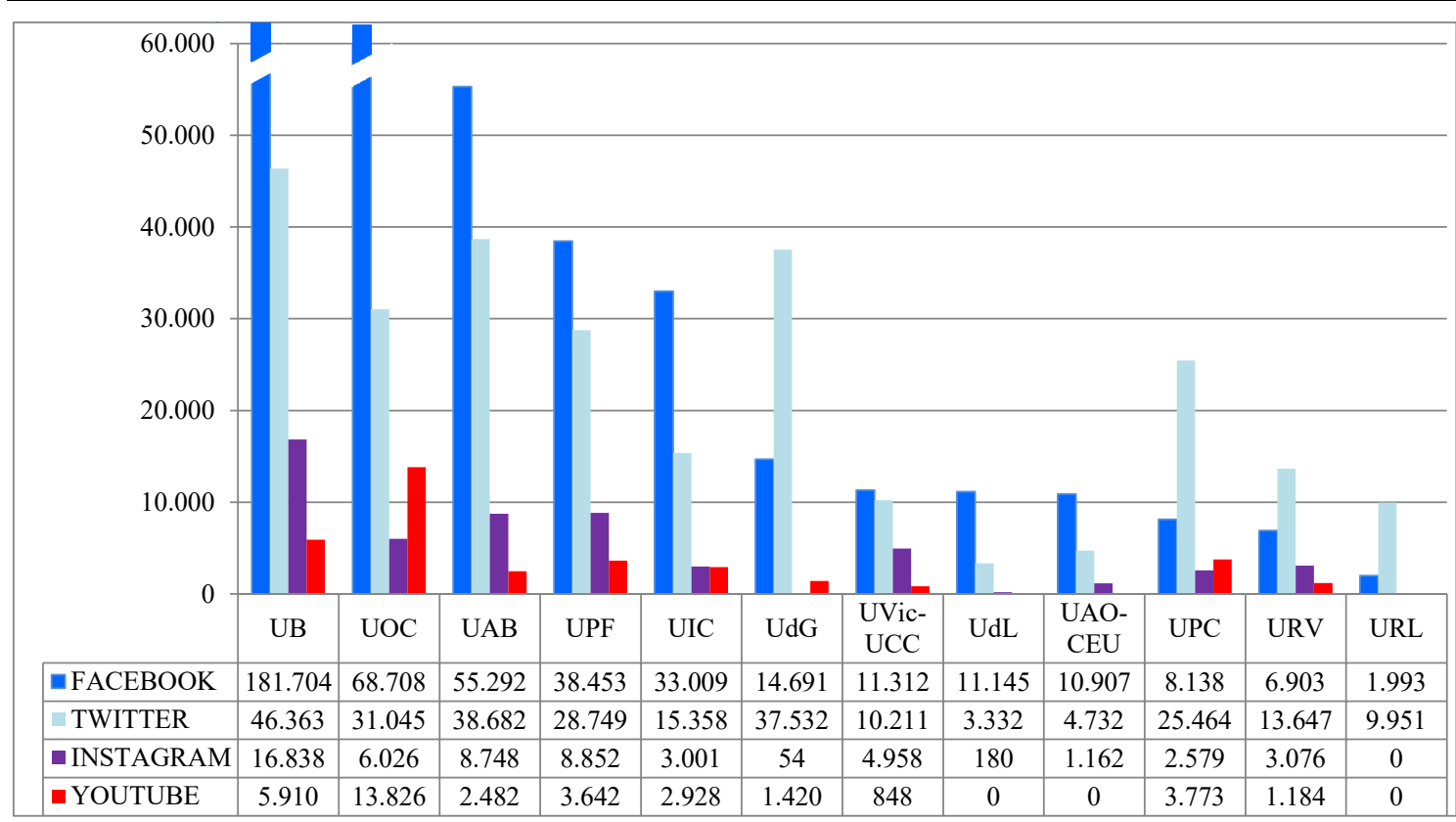

Fuente: Elaboración propia.

La UB era, con destacable diferencia, la universidad más seguida en Facebook (Figura 1), con más del doble de seguidores que la inmediatamente posterior, la UOC y a continuación se situaba la UAB. En Twitter también era la UB la más destacada, mientras que la UAB y la UdG ocupaban el segundo y tercer lugar respectivamente. 
Con respecto a Instagram, la UB era también la más seguida de manera destacada doblando prácticamente en seguidores a la UPF y la UAB, las siguientes en número de seguidores. Las universidades menos seguidas en Instagram en el momento del análisis eran la UdL, la UdG y la URL, que no tenía perfil. En YouTube, la UOC lideraba el listado de universidades siendo la más seguida, con más del doble de seguidores que la UB, en segunda posición, y más del triple que la UPC, en tercera posición. Al ser la UOC una universidad a distancia trabaja mucho más el uso de YouTube que las otras universidades, como se explica en el apartado de publicaciones, y en consecuencia tiene muchos más seguidores en esta red que el resto. Parece que las universidades UAO-CEU, UdL y URL no tenían seguidores por tener la cifra de seguidores oculta, aunque sí que tenían perfil en la red.

En concreto, analizando las cuatro RRSS, la UB era la universidad con más seguidores en Facebook, Twitter e Instagram, y la segunda en YouTube. La posición dominante en número de seguidores de la UB en valor absoluto responde a que es también la universidad con más número de estudiantes de Cataluña. En Facebook y Twitter también destacaban la UAB y la UOC, y en Instagram y YouTube la UOC y la UPF. Sus posiciones elevadas pueden explicarse, al igual que en el caso de la UB, porque se sitúan entre las universidades con más estudiantes de Cataluña. Además, la UOC, al ser una universidad virtual, tiene alumnos no solo de España sino de muchos otros países. Por el contrario, la URL era la universidad con menos seguidores en redes, situándose en último lugar en Facebook y sin seguidores en Instagram ni en YouTube.

\section{Figura 2}

Seguidores totales de las universidades en cada red social

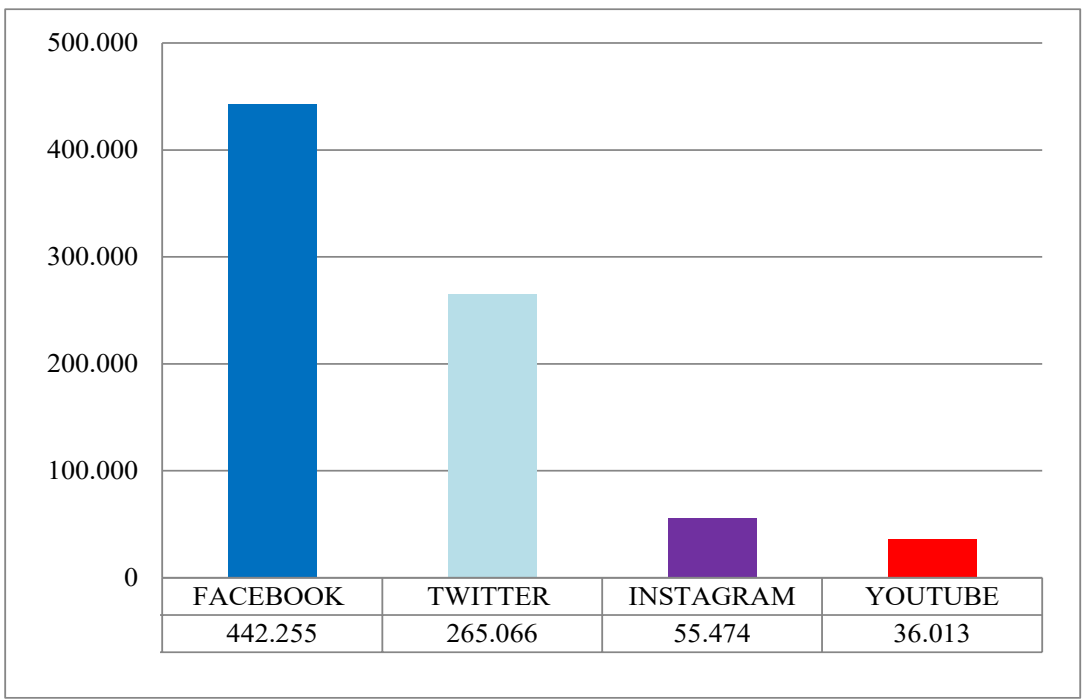

Fuente: Elaboración propia.

La Figura 2 muestra los datos agregados de seguidores de todas las universidades correspondientes a cada red social. Se observa que Facebook era la red social que, con gran diferencia, reunía el mayor número de seguidores. De hecho, esta red social contaba con 85.702 más seguidores que las otras tres sumadas. Twitter era la otra red social de mayor importancia en seguidores. Instagram y YouTube tenían un número de seguidores mucho más discreto o insignificante respecto a las anteriores RRSS. 


\subsubsection{Seguidores en las RRSS en valor relativo}

En la Figura 3 se muestra el número de seguidores en Facebook, Twitter, Instagram y YouTube en valor relativo por el número de estudiantes de cada universidad.

Las universidades más seguidas en Facebook (Figura 3) eran la UIC y la UAO-CEU, y la UB se situaba en tercera posición. La universidad con menos seguidores en esta red social era la URL. En valores relativos la universidad con el liderazgo en Twitter era la UIC, después se situaban la UAO-CEU y la UdG. La universidad con menos seguidores en Twitter era la UdL.

Con respecto a Instagram, las universidades más seguidas eran la UIC, la UAO-CEU y la UVicUCC, que son todas universidades privadas. Las universidades con menos seguidores en Instagram eran la UdG, la UdL y la URL. En cuanto a YouTube, las universidades con más seguidores por estudiante eran la UIC, la UOC y la UPF. Parece que las universidades UAOCEU, UdL y URL no tuvieran seguidores porque estos datos en YouTube los tenían ocultos, por lo que no se pueden valorar. De este modo, si no se tienen en cuenta estas universidades, la menos seguida era la UAB.

\section{Figura 3}

Seguidores por estudiante de cada universidad en las RRSS

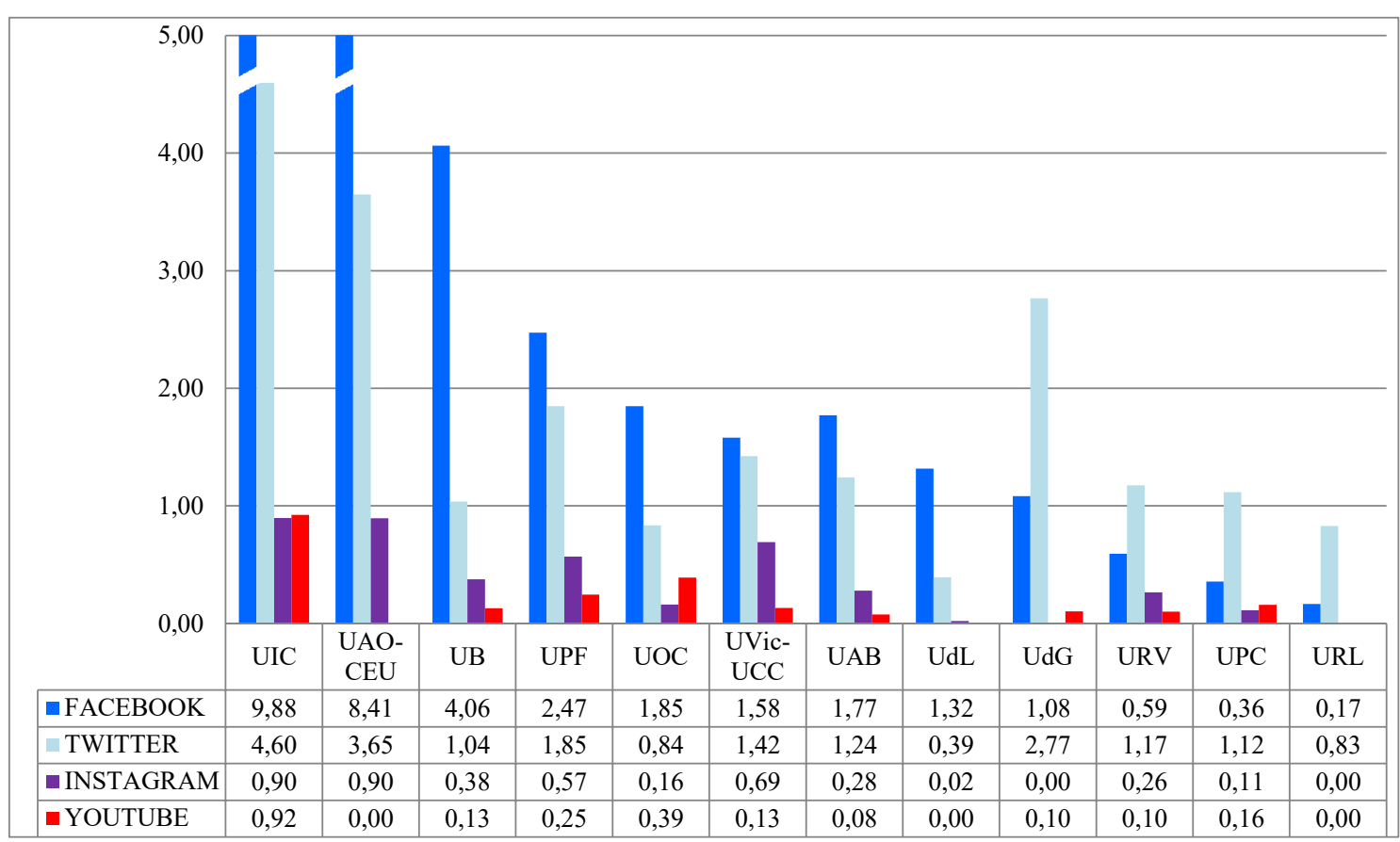

Fuente: Elaboración propia.

En síntesis, cuando se pondera el número de seguidores en las redes por el número de estudiantes, la UIC pasaba a ser la universidad que tenía más seguidores en relación con los estudiantes en todas las RRSS. En segundo lugar se encontraba la UAO-CEU en las redes de Facebook, Twitter e Instagram, aunque en YouTube no se conocía su número de seguidores. Ambas universidades son privadas. Por el contrario, la URL era la universidad menos seguida en las diferentes RRSS. 
Es interesante destacar que, en valor absoluto, las universidades públicas ocupaban las primeras posiciones, posiblemente debido a la influencia del gran número de estudiantes que tienen, pero, en valor relativo, las universidades privadas eran las que sobresalían pues tanto la UIC como la UAO-CEU son universidades privadas.

\subsubsection{Aumento de los seguidores de las universidades}

En este apartado se ha comparado el número de seguidores que tenían las universidades de Cataluña en las RRSS en dos momentos diferentes para analizar sus variaciones: en la semana del 15-21 de febrero de 2018 y en la semana del 20-26 de junio de 2018 (Figuras 4 y 5). Es decir, se estudia el aumento de seguidores, no el número superior o inferior de seguidores que tienen las universidades, que ya fue tratado en el apartado anterior.

El primer resultado observable es que todas las universidades aumentan su número de seguidores en las diferentes RRSS. En Facebook (Figura 4) se aprecia que la universidad que más crecimiento tuvo fue la UB, seguida con un valor cuatro veces inferior por la UOC, después se situaba la UAB. La universidad con menos crecimiento era la URL. En Twitter, la universidad con más aumento fue la UAB, seguida de cerca por la UB, después se situaba la UPF, y la URL repetía como la universidad con menos aumento.

\section{Figura 4}

Variación de los seguidores de cada universidad en las redes Facebook y Twitter

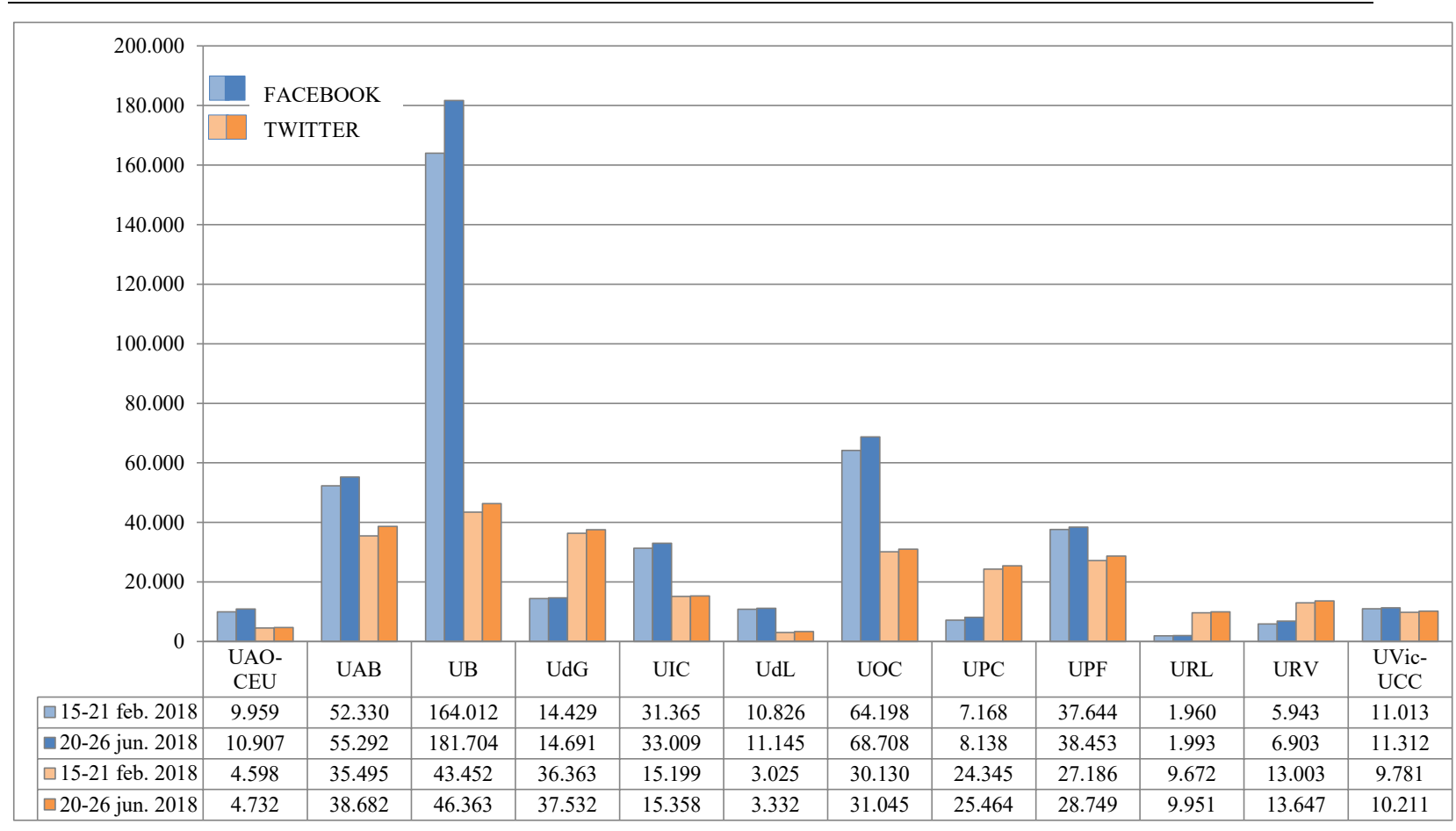

Fuente: Elaboración propia.

En Instagram (Figura 5), la universidad que más aumentó su nivel de seguidores fue la UB, le seguía la UAB y a gran distancia la UPF. Las universidades con menos aumento fueron la UdL y la URL (que no tenía seguidores). En YouTube, la universidad que más creció en seguidores fue la UOC, le seguía la UB y la UIC. La universidad que menos creció fue la UVic-UCC. Las 
universidades UAO-CEU, UdL y URL no se pueden valorar porque tenían el número de seguidores oculto en esta red social.

En definitiva, la universidad que más aumentó sus seguidores en las diferentes RRSS durante el periodo analizado fue la UB, situada en la primera posición en Facebook e Instagram y en segunda posición en Twitter y YouTube. Al igual que en los seguidores en valor absoluto, el hecho de ser la universidad de Cataluña con más estudiantes explica en gran medida esta situación dominante de la UB. Por contra, las universidades que experimentaron menos crecimiento fueron la URL y la UdL.

\section{Figura 5}

Variación de los seguidores de cada universidad en las redes Instagram y YouTube

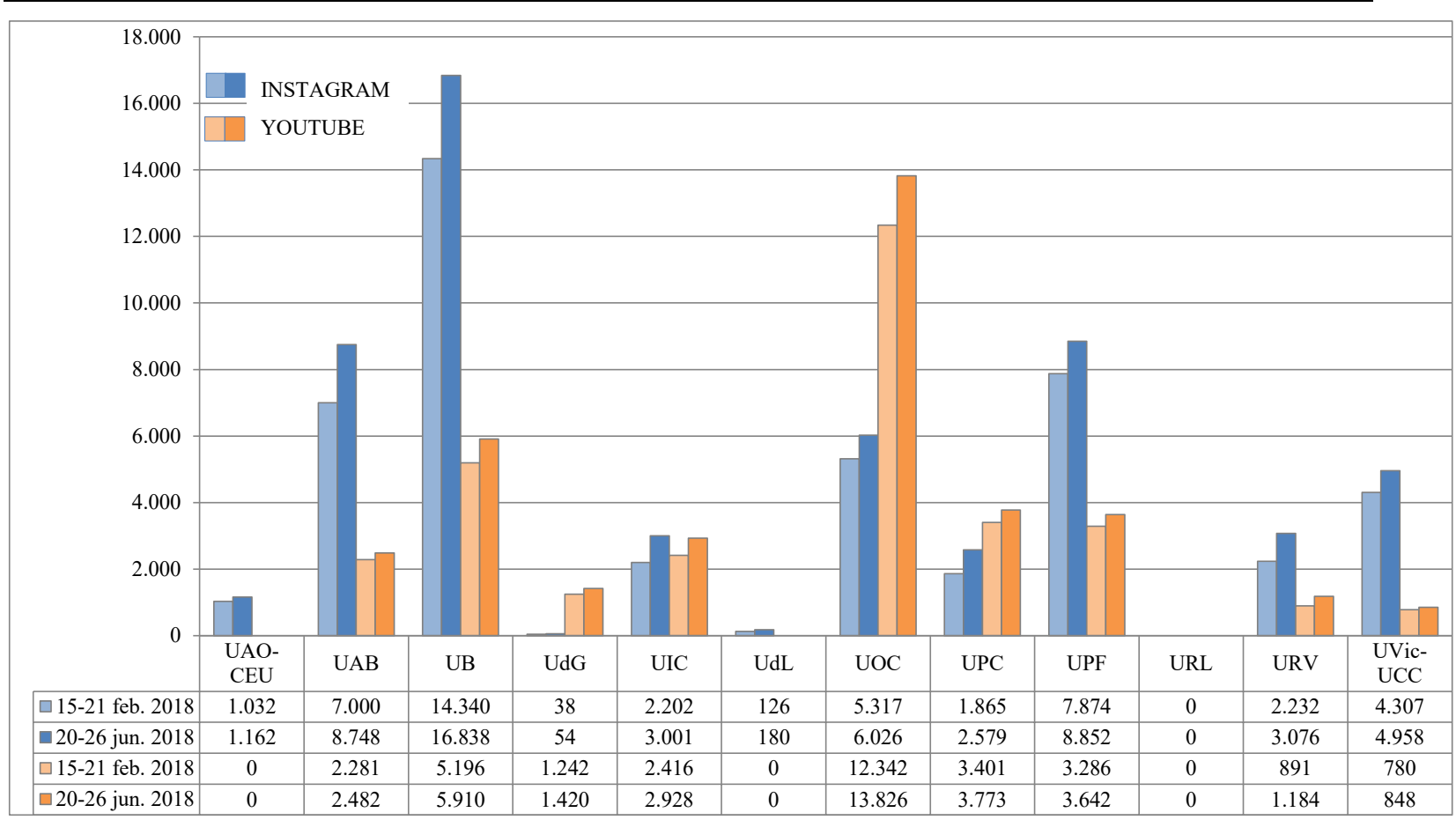

Fuente: Elaboración propia.

\subsection{Publicaciones en las RRSS}

Los resultados del número de publicaciones que hicieron las universidades en sus perfiles de las RRSS, del día 20 al 26 de junio, se han representado en la Figura 6.

En Facebook (Figura 6) la universidad con mayor frecuencia de publicación durante el período fue la UOC, seguida de la UVic-UCC, la URV y, de forma decreciente, el resto de las universidades. La UOC es una universidad a distancia por lo que publica mucho más en YouTube que las otras universidades para compensar su no presencialidad. La universidad con menos publicaciones fue la UdG. En la red social de Twitter las publicaciones se disparan en prácticamente todas las universidades. En esta red la universidad que tenía más frecuencia de publicación fue la UAB, la siguiente fue la UOC, y en tercera posición la UdG. La universidad con menos publicaciones era la UdL. 
Instagram y YouTube tenían unos niveles de publicación muy inferiores a Facebook o Twitter. En Instagram, la universidad con mayor frecuencia de publicación era la UVic-UCC, la siguiente era la UOC y en tercera posición se encontraba la UAB. Las universidades sin publicaciones durante el periodo estudiado fueron la UdL, la URL y la UdG. En YouTube las publicaciones consisten en colgar vídeos. Así, la universidad que colgó más vídeos fue la UOC, la siguiente la UB, y en tercera posición se situaba la UdG. Las universidades que no colgaron ningún vídeo fueron la UdL, la URL, la UAB y la UPC.

En síntesis, a nivel de publicaciones destacó la UOC como la universidad que más publicó en las diferentes RRSS, ocupando la primera posición en Facebook y YouTube respecto al resto de universidades y la segunda posición en Instagram y Twitter. Al ser la UOC una universidad a distancia publica más que las otras universidades para mantener una comunicación con sus estudiantes que no tiene mediante el campus presencial. Por lo que respecta a la regularidad, le seguía la UVic-UCC, en primera y segunda posición en Instagram y Facebook y en cuarta posición en Twitter y YouTube. Se debe comentar el caso de la UdG, que no es un caso aislado pero si el más relevante, mal posicionada con respecto al resto de universidades en cuanto a sus publicaciones en Facebook e Instagram, en contraposición al buen posicionamiento que conseguía en Twitter y YouTube. Este hecho demuestra que las universidades se concentran solo en una o dos RRSS y no siguen una estrategia unificada para todas. La URL y la UdL realizaron un número escaso o nulo de publicaciones en las RRSS estudiadas.

\section{Figura 6}

Número de publicaciones de cada universidad en las RRSS

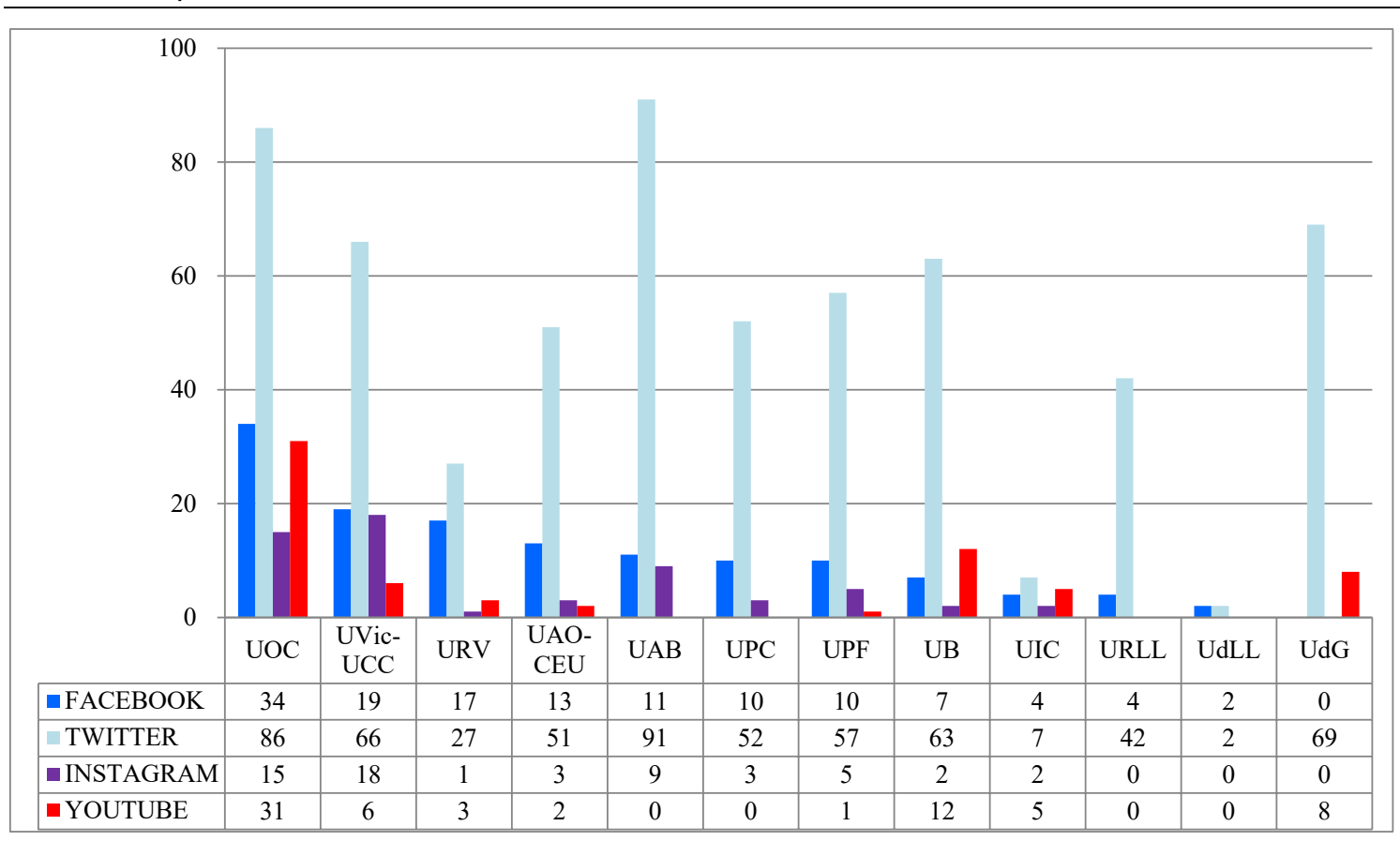

Fuente: Elaboración propia.

A partir de la suma de las publicaciones realizadas por las diferentes universidades de Cataluña durante el periodo analizado (Figura 7) destacó Twitter como la red social donde más publicaban las universidades catalanas, muy por encima del resto de RRSS, representando el $70 \%$ de las 
publicaciones totales. La siguiente red social de publicación era Facebook y le seguían, en valores muy inferiores, YouTube e Instagram en este orden.

\section{Figura 7}

Número total de publicaciones de las universidades en cada red social

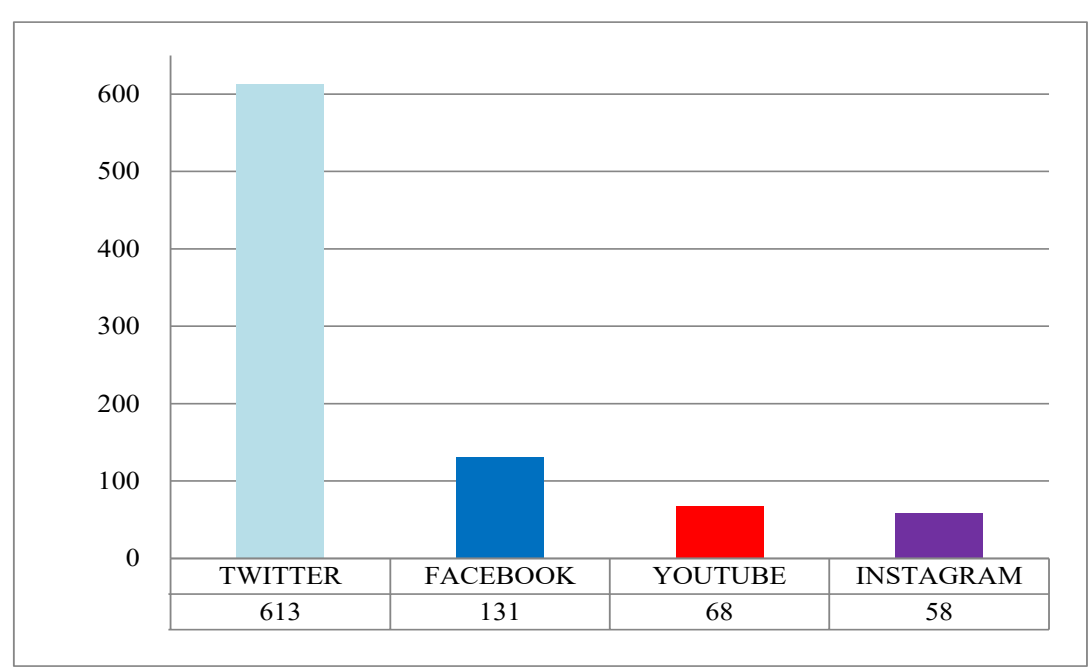

Fuente: Elaboración propia.

\subsection{Reacciones "Me gusta" a las publicaciones}

\subsubsection{Reacciones "Me gusta" a las publicaciones en valor absoluto}

Las reacciones a las publicaciones, medidas en número de "Me gusta", que tuvieron las universidades en las distintas RRSS se han representado en la Figura 8.

En Facebook, (Figura 8) la UB era la universidad que conseguía más "Me gusta" prácticamente doblando a la siguiente universidad, la UPF, y les seguía la URV. En cambio, la UdG no tenía ningún "Me gusta”. En Twitter la UPF se situaba en primera posición, seguida de cerca por la UAB y la UPC. La UdL no tenía reacciones en esta red.

Con respecto a Instagram, la UAB era la universidad que obtenía más interacción a una distancia de más de 1.000 "Me gusta" de la UVic-UCC, situada en segunda posición, les seguía la UPF. Las universidades sin reacciones eran la UdG, UdL y URL. En YouTube, las universidades con más "Me gusta" eran la UOC y la UIC, mientras que la UB se situaba en tercera posición. En esta red las universidades sin interacción eran muy numerosas: UAO-CEU, UAB, UdL, UPC, UPF y URL, la mayoría de ellas porque tampoco colgaron vídeos.

En conclusión, las primeras posiciones de las reacciones de los usuarios en valor absoluto son ocupadas por las universidades con más estudiantes de Cataluña, como ya sucedía con el número de seguidores. Así, la UB y la UAB se situaban en primera posición en Facebook e Instagram respectivamente. En Twitter la UPF se situaba en primera posición, y en YouTube lideraba la UOC, al ser una universidad virtual y publicar mucho en esta red además de su elevado número de estudiantes. Es interesante destacar que la UPF fue la universidad que mantuvo un nivel de "Me gusta" elevado más regular en las RRSS, manteniéndose entre las 
tres primeras universidades en las reacciones de las diferentes RRSS, excepto en YouTube donde no tuvo reacciones. También cabe destacar las posiciones elevadas de la UVic-UCC y la UIC en Instagram y YouTube respectivamente, a pesar de su reducido número de estudiantes. Las otras universidades no seguían un patrón concreto porque la interacción que recibían se presentaba muy variable de una red social a otra en las que tenían presencia. La UdL destacaba en negativo por ser una de las universidades con menos interacción.

\section{Figura 8}

Reacciones "Me gusta" de cada universidad en RRSS

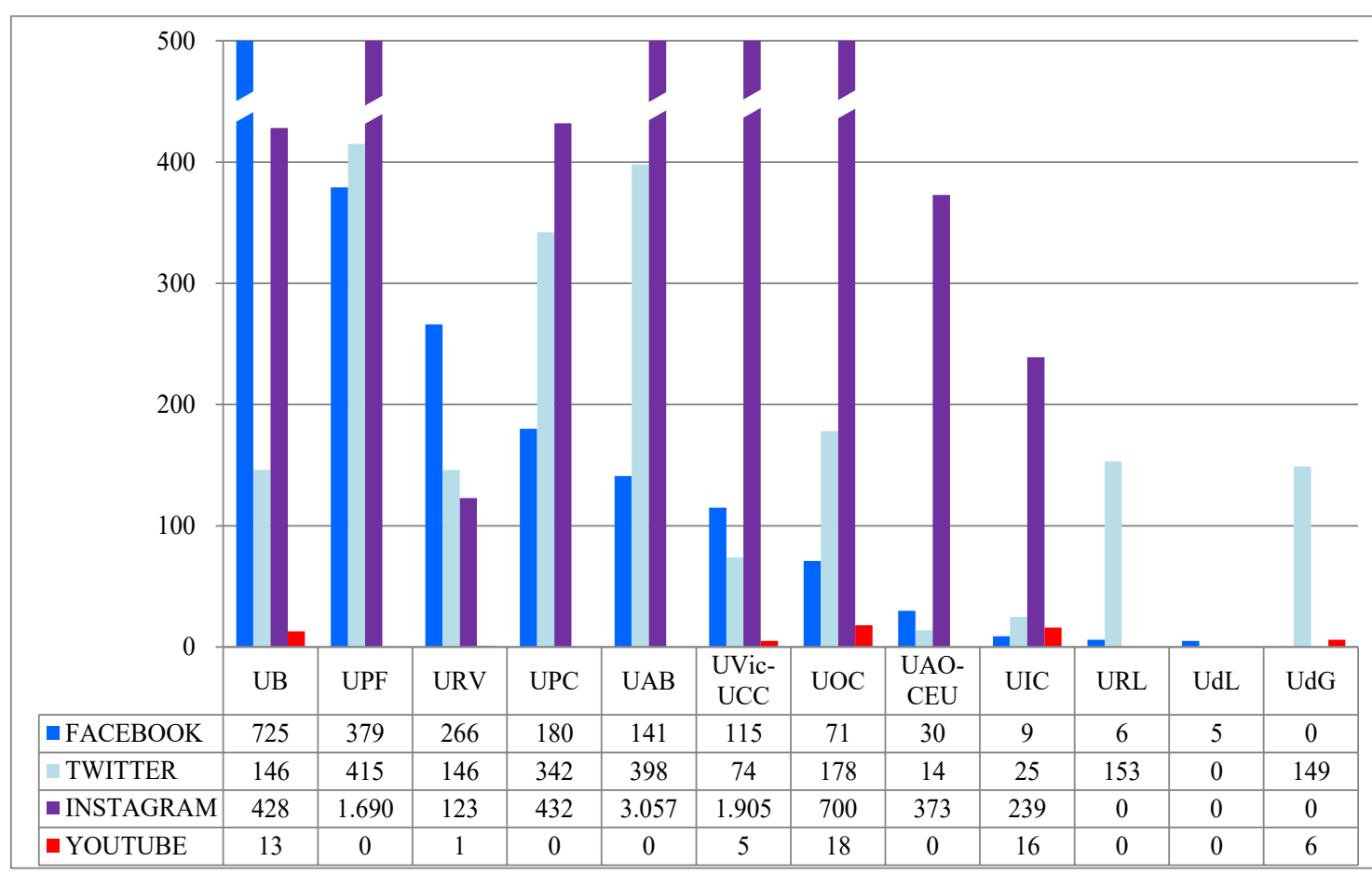

Fuente: Elaboración propia.

\section{Figura 9}

Número total de reacciones "Me gusta" de las universidades en cada red social

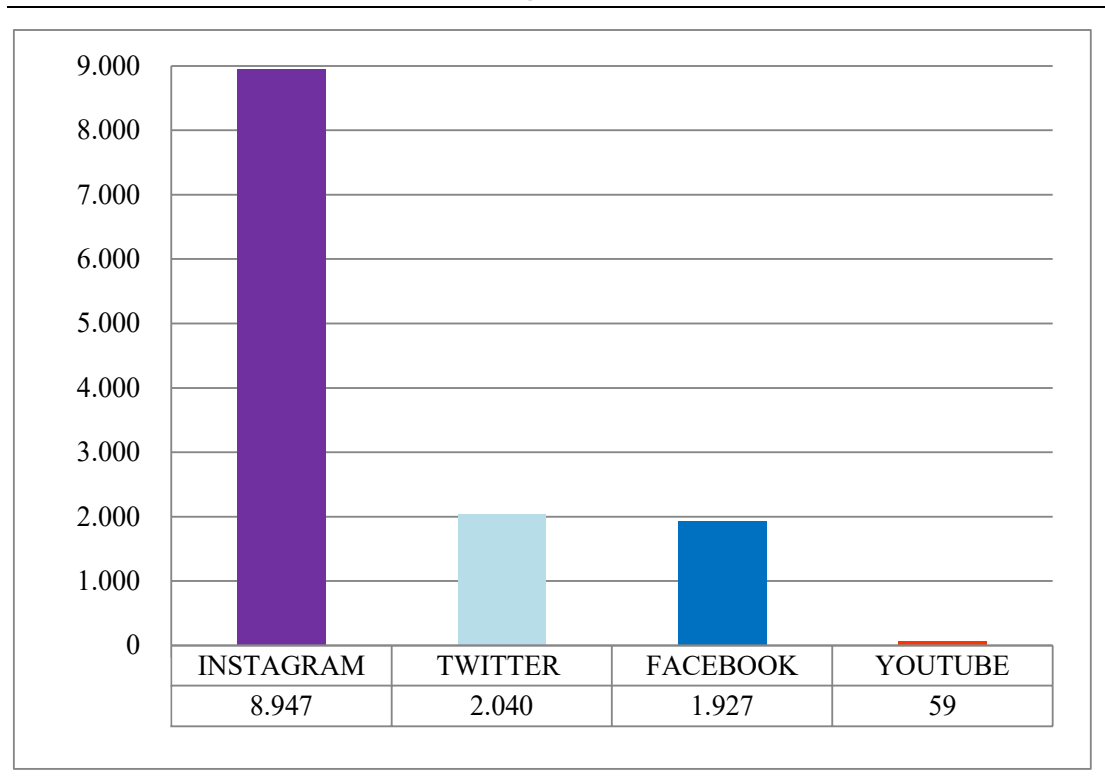

Fuente: Elaboración propia. 
En el total de "Me gusta" sumados (Figura 9) en las diferentes RRSS, Instagram destacaba como la red social que recibía más interacción. Esta red social multiplicaba por cuatro el valor de "Me gusta" de Twitter o Facebook, que eran similares entre ellos. YouTube recibió mucha menos interacción que las otras redes. Tiene sentido que YouTube fuera la red con menor respuesta, ya que también era una red con pocas publicaciones. No obstante, destaca que Instagram fuera la red con menor número de publicaciones y que obtuviera un nivel de reacciones muy por encima de las otras RRSS.

\subsubsection{Reacciones "Me gusta" a las publicaciones en valor relativo}

Seguidamente se analizan los "Me gusta" relativos por el número de estudiantes de cada universidad (Figura 10). En Facebook, en valor relativo, la UPF era la que recibía más "Me gusta", le seguían a poca distancia la UAO-CEU y la URV. La UdG no recibió ningún "Me gusta". En Twitter, la UPF repetía como la universidad que recibía más interacción prácticamente doblando a la siguiente universidad, la UPC. En tercera posición les seguía la UAB. En cambio, la UdL no consiguió ningún "Me gusta".

En Instagram las universidades que recibían más "Me gusta", en valor relativo, eran la UAOCEU y la UVic-UCC, en primera y segunda posición respectivamente. Con menos de la mitad de "Me gusta" recibidos les seguía la UPF. Las universidades sin reacciones de los usuarios eran la UdG, la UdL y la URL. Con respecto a YouTube la universidad que generó más "Me gusta", en valor relativo, a gran distancia de las otras, fue la UIC. La siguiente universidad, con un valor seis veces inferior, era la UVic-UCC y le seguía la UOC. Las universidades sin interacción fueron la UAO-CEU, la UAB, la UdL, la UPC, la UPF y la URL.

\section{Figura 10}

Reacciones "Me gusta" por cada 1.000 estudiantes de cada universidad en RRSS

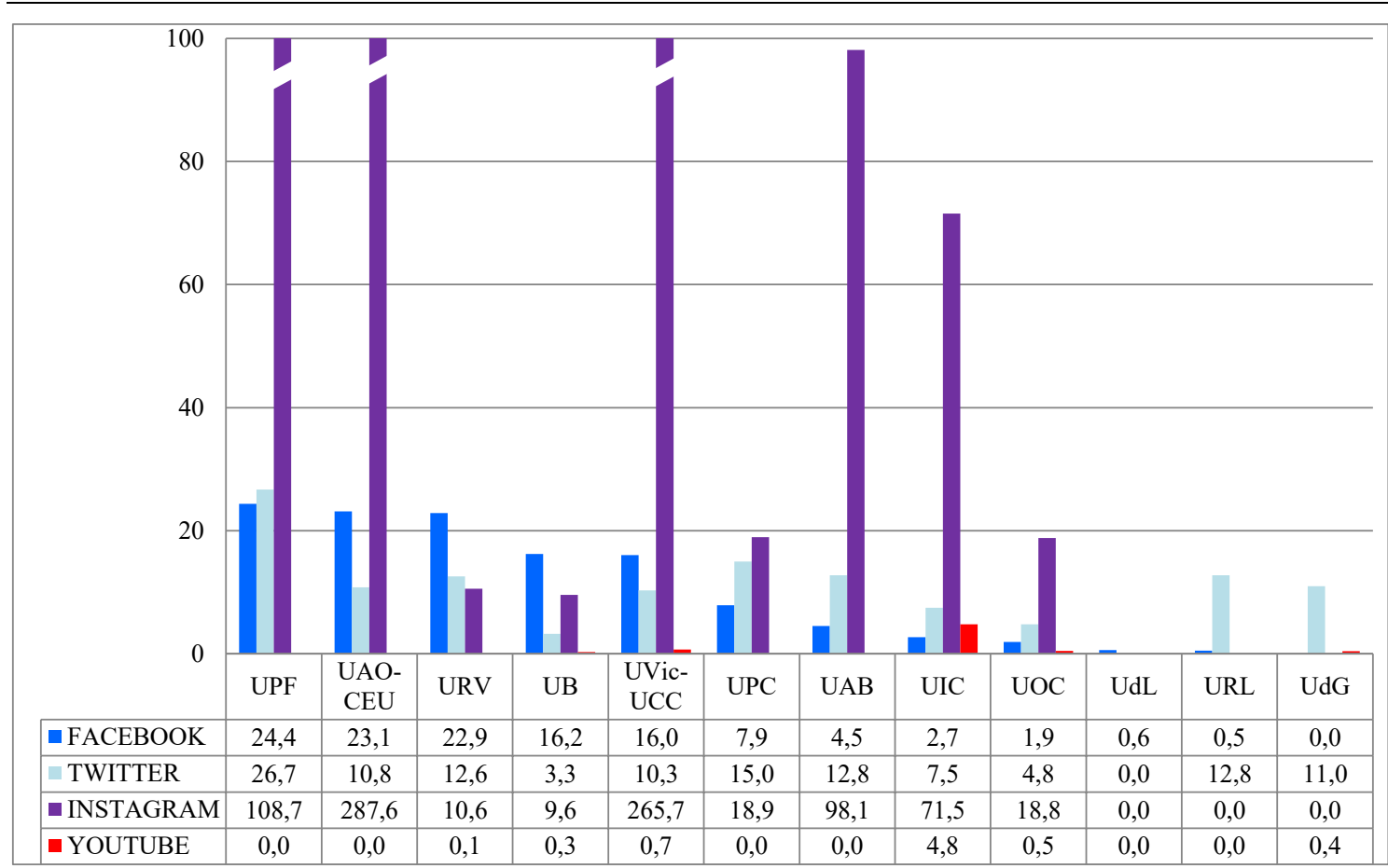

Fuente: Elaboración propia. 
En síntesis, en las reacciones en valor relativo, la UPF se situaba claramente en primera posición en Facebook y en Twitter, respecto a la reacción de los usuarios, y en tercera posición en Instagram, aunque sin reacciones en YouTube. La UVic-UCC era la otra universidad que mantenía una regularidad de "Me gusta" elevada en valor relativo. La UAO-CEU también tenía una posición elevada en reacciones en las diferentes redes, aunque en YouTube no tenía reacciones. Es interesante resaltar que al trabajar en valor relativo las universidades públicas, exceptuando el caso de la UPF, descendían y cedían las primeras posiciones a las universidades privadas que escalaban desde sus posiciones inferiores en valor absoluto a las primeras. En negativo, destacó la UdL sin reacciones en Instagram, Twitter o YouTube y sólo con interacciones en Facebook. La otra universidad que también destacó por pocas interacciones fue la UdG.

\section{Discusión y conclusiones}

En el presente trabajo se estudiaron los perfiles en RRSS de las universidades catalanas. Globalmente se concluye que la red social con más seguidores era Facebook y le seguía Twitter con la mitad de seguidores (comparativamente Instagram y YouTube tenían un nivel de seguidores muy inferior en el conjunto de las universidades). Al observar las publicaciones de las distintas redes, Twitter absorbía el $70 \%$ de las publicaciones que realizaban las universidades en todas las RRSS. Instagram destacaba obteniendo la mayor representación de reacciones "Me gusta" de los usuarios por sus publicaciones, siendo la tercera red social en seguidores y la última en publicaciones.

Los resultados muestran que las universidades de Cataluña utilizan las RRSS de forma muy variada, sin una comunicación y planificación digital unificada para todas. Aun así, el trabajo desveló que algunas universidades obtuvieron buenos resultados en RRSS en determinadas variables y en cambio otras se situaron en la parte final de todas las variables.

En valor absoluto, en número de seguidores sobresalía la UB y también la UAB, la UOC y la UPF, en aumento de seguidores en los dos periodos analizados la UB volvía a destacar como la universidad que más creció. La fuerte dominación de la UB se explica porque es la universidad con más estudiantes de Cataluña, así como las posiciones elevadas de las otras universidades que la acompañan pues también son las universidades con más estudiantes. Además, en el caso de la UOC es una universidad virtual con alumnos de muchos países. La UPF y la UB destacaban en número de reacciones "Me gusta". Sin embargo, todas ellas mantenían una posición intermedia en publicaciones donde destacaban la UOC y la UVic-UCC. Resaltan las buenas posiciones de la UPF siendo la $5 \underline{a}$ universidad en número de seguidores por debajo de la UPC y no muy lejos de los estudiantes de otras universidades como la UdG. Por contra, la UdL y URL se situaban en todas las variables analizadas en las diferentes redes en posiciones inferiores.

En valor relativo, cuando se pondera el número de seguidores en las redes por el número de estudiantes, destacaba la UIC como la universidad con más seguidores seguida de la UAO-CEU, ambas privadas desplazando a las públicas de estas primeras posiciones. Por lo que respecta a la reacción de los usuarios en valor relativo, sobresalían la UPF y la UVic-UCC y les seguiría la UAO-CEU si no se tuviera en cuenta YouTube. En cuanto a públicas, la UPF lideraba en reacciones, pero también tenía una posición elevada en número de seguidores en valor relativo por lo que dentro de las universidades públicas es una excepción. Así en valor relativo, considerando su número de estudiantes, la UPF es la que mejores resultados obtiene y la que mejor trabaja las RRSS, pudiendo ser un referente. Entre las universidades privadas hay varias que 
obtenían los mejores resultados y se podría deducir que trabajan mejor sus redes. En primer lugar, la UVicUCC destacaba en reacciones y en publicaciones y mantenía una posición intermedia en seguidores. En segundo lugar, la UAO-CEU sobresalía en seguidores y reacciones, si no se tiene en cuenta YouTube, y tenía un perfil bajo en publicaciones. Por último, la UIC destacaba en seguidores, a pesar de posiciones leves en publicaciones y reacciones. Así, quizás sería la UVic-UCC la que por su consistencia en las tres variables analizadas podría considerarse un referente para las privadas. En sentido contrario, a nivel de los seguidores en valores relativos y absolutos, la UdL y la URL repetían como las universidades con los valores más bajos, y en reacciones esta posición era para la UdL y la UdG.

La investigación estudia el uso de las RRSS de las universidades públicas y privadas en un mismo análisis para poder comparar sus actuaciones. En este sentido, el estudio revela que en valor absoluto las universidades públicas se sitúan en las primeras posiciones, ayudadas por el gran número de estudiantes que tienen. En cambio, en valor relativo destacan las privadas, con la excepción de la pública UPF que sobresalía en reacciones y la URL que, siendo privada, tenía posiciones inferiores. De este modo, la investigación muestra que en general, aunque en valor absoluto destacaban las públicas, al corregir por número de estudiantes, las privadas obtenían mejores resultados. Consecuentemente se podría afirmar que las privadas están trabajando mejor las RRSS que las públicas y que estas deberían reorientar su trabajo en redes.

En general, los resultados parecen indicar una falta de planificación y estrategia bien elaboradas detrás del uso de las RRSS por parte de las universidades catalanas. Se observa un uso muy desigual de estas RRSS entre universidades (mientras algunas obtienen resultados muy destacados, otras repiten en posiciones bajas en las variables analizadas). Además, las universidades no tienen una constancia de actuación o resultados en las diferentes redes, concentran su actuación en una o dos redes, destacando sólo en ellas y obtienen valores muy bajos en las otras. Se debe tener en cuenta que las actuaciones adaptadas y diferentes en cada red son recomendables, pero debería evitarse trabajar solamente una o dos RRSS y abandonar las otras.

El presente artículo, centrado en las universidades de Cataluña, coincide con las conclusiones sobre la poca planificación de las RRSS y el marketing digital de las universidades en España a las que llegaron otros autores (Blázquez et al. 2020; Paniagua Rojano y Gómez Calderón, 2012; Reina Estévez et al., 2012; Simón Onieva, 2015; Zarco et al., 2016). Por esta razón es imprescindible que las universidades modifiquen sus estrategias de comunicación dando más protagonismo a las estrategias de marketing digital y RRSS y que planifiquen sus actuaciones en las RRSS (San Millán Fernández et al., 2008), especialmente las universidades públicas. No obstante, en el uso de las RRSS se ha identificado unas universidades que obtienen buenos resultados y que pueden ser un referente: la UPF para las universidades públicas y la UVic-UCC para las universidades privadas.

Este trabajo aporta valor al ser uno de los primeros en analizar la actividad y los resultados en las RRSS de las universidades de Cataluña en el momento de preinscripción universitaria. Por ello, puede servir de referencia para la reorientación de las estrategias de comunicación de las universidades. Se ha trabajado con las cuentas principales de las universidades en RRSS para homogeneizar y simplificar el estudio. No obstante, sería de interés en futuros artículos estudiar y comparar los perfiles en RRSS de las facultades y campus que tienen las universidades. También se podrían estudiar por separado las universidades públicas y privadas, aunque aquí se hayan estudiado a la vez al competir en un mismo mercado. En futuras líneas de investigación, sería de interés comparar este estudio con otras comunidades autónomas de España, así como con estudios posteriores en el tiempo de la misma comunidad autónoma. Por último, se podría 
ampliar este trabajo investigando la red social de Linkedln o estudiando otras herramientas de comunicación que utilizan las instituciones de educación superior.

\section{Referencias}

Amaral, I., y Santos, S. (2020). Social networks and institutional communication: The case of Portuguese universities. Revista prisma social, 32, 20-45.

https://dialnet.unirioja.es/descarga/articulo/7263736.pdf

Ashley, C., y Tuten, T. (2015). Creative Strategies in Social Media Marketing: An Exploratory Study of Branded Social Content and Consumer Engagement. Psychology \& Marketing, 32(1), 15-27. https://doi.org/10.1002/mar.20761

Bélanger, C. H., Bali, S., y Longden, B. (2014). How Canadian universities use social media to brand themselves. Tertiary Education and Management, 20(1), 14-29.

https://doi.org/10.1080/13583883.2013.852237

Benedicto, J., Echaves, A., Jurado, T., Ramos, M., y Tejerina, B. (2017). Informe Juventud en España 2016. http://www.injuve.es/sites/default/files/2017/24/publicaciones/informe-juventud-2016.pdf

Blázquez, F., Rodríguez, C., y Teijeiro, M. (2020). A new era of communication in Higher Education. Facebook as a marketing tool. Espacios, 41(6), 23.

https://www.revistaespacios.com/a20v41n06/a20v41n06p23.pdf

Brito, J. G., Laaser, W., y Toloza, E. A. (2012). El uso de redes sociales por parte de las universidades a nivel institucional. Un estudio comparativo. RED Revista de educación a distancia, 32, 1-38.

https://revistas.um.es/red/article/view/233071

Carmelo García, M. (2007). Marketing universitario: conceptos y herramientas de marketing aplicadas a la educación superior en España [Tesis doctoral, Universidad Autónoma de Madrid]. http://hdl.handle.net/10486/2392

De Vries, L., Gensler, S., y Leeflang, P. S. H. (2012). Popularity of Brand Posts on Brand Fan Pages: An Investigation of the Effects of Social Media Marketing. Journal of Interactive Marketing, 26(2), 8391. https://doi.org/10.1016/j.intmar.2012.01.003

Egan, J. (2011). Relationships Marketing: Exploring Relational Strategies in Marketing. Prentice Hall.

Freire, J. (2008). Los retos y oportunidades de la web 2.0 para las universidades. En R. Jiménez Cano y F. Polo (eds.), La gran guía de los blogs 2008 (pp. 82-90). El Cobre.

Fundación Telefónica. (2016). La Sociedad de la Información en España 2016. Editorial Ariel; Fundación Telefónica. https://www.fundaciontelefonica.com/cultura-digital/publicaciones/la-sociedad-dela-informacion-en-espana-2016/558/

Fundación Telefónica. (2020). Sociedad digital en España 2019. Penguin Random House. 
García Orosa, B. (2009). Gabinetes de comunicación online: claves para generar información corporativa en la red. Comunicación social.

Generalitat de Catalunya. (2017). Informe de la preinscripció universitària a Catalunya. http://universitats.gencat.cat/ca/altres_pagines/informe_i_estadistiques/informes_i_estad_pre/

Generalitat de Catalunya. (2019). Preinscripción universitaria. https://web.gencat.cat/es/tramits/tramitstemes/Preinscripcio-universitaria

Hayes, T. J. (1993). Image and the University. Journal of Marketing for Higher Education, 4(1-2), 423-425. https://doi.org/10.1300/j050v04n01_28

IAB Spain Research. (2018). Estudio anual de redes sociales 2018. https://iabspain.es/estudio/estudioanual-de-redes-sociales-2018/

Kimmons, R., Veletsianos, G., y Woodward, S. (2017). Institutional Uses of Twitter in U.S. Higher Education. Innovative Higher Education, 42(2), 97-111. https://doi.org/10.1007/s10755-016-9375-6

Lewison, D. M., y Hawes, J. M. (2007). Student Target Marketing Strategies for Universities. Journal of College Admission, 196, 14-19. http://files.eric.ed.gov/fulltext/EJ783948.pdf

Mazzarol, T., y Soutar, G. N. (2002). «Push-pull» factors influencing international student destination choice. International Journal of Educational Management, 16(2), 82-90. https://doi.org/10.1108/09513540210418403

Ministerio de Ciencia Innovación y Universidades. (2019). Estadísticas e indicadores universitarios. Estadistica de estudiantes.

http://www.ciencia.gob.es/portal/site/MICINN/menuitem.7eeac5cd345b4f34f09dfd1001432ea0/ ?vgnextoid=0930dd449de8b610VgnVCM1000001d04140aRCRD

Ministerio de Educación Cultura y Deporte. (2016). Datos y cifras del sistema universitario español. Curso 2015/2016. Secretaría General de Universidades.

https://sede.educacion.gob.es/publiventa/datos-y-cifras-del-sistema-universitario-espanol-curso20152016/estadisticas-universidad-espana/21461

Onieva, J. E. S. (2017). Uso de las Redes Sociales por las universidades públicas andaluzas. Análisis de contenido de la cuenta oficial de Twitter. Estudios sobre el mensaje periodístico, 23(1), 631-645. https://doi.org/10.5209/ESMP.55618

Paniagua Rojano, F. J., y Gómez Calderón, B. J. (2012). Hacia la comunicación 2.0. El uso de las redes sociales por parte de las universidades españolas. ICONO14 Revista de comunicación y tecnologías emergentes, 10(3), 346-364. https://doi.org/10.7195/ri14.v10i3.473

Paniagua Rojano, F. J., Gómez Calderón, B. J., y Fernández Sande, M. (2012). La incorporación de los departamentos de comunicación de las universidades españolas al entorno digital. Un análisis cuantitativo. Estudios sobre el mensaje periodístico, 18, 691-701.

https://doi.org/10.5209/rev_ESMP.2012.v18.40948 
Reina Estévez, J., Fernández Castillo, I., y Noguer Jiménez, Á. (2012). El Uso de las Redes Sociales en las Universidades Andaluzas: El Caso de Facebook y Twitter. Revista internacional de relaciones públicas, 2(4), 123-144. https://doi.org/10.5783/RIRP-4-2012-06-123-144

Rissoan, R. (2006). Redes sociales: comprender y dominar las nuevas herramientas de comunicación. ENI.

Rodríguez Ruibal, A., y Santamaría Cristino, P. (2012). Análisis del uso de las redes sociales en Internet: Facebook y Twitter en las Universidades españolas. Icono14, 10(2), 228-246. https://doi.org/10.7195/ri14.v10i2.198

San Millán Fernández, E., Blanco Jiménez, F. J., y Arco Prieto, J. C. del. (2008). Comunicación corporativa 2.0 en la Universidad Rey Juan Carlos. En E. J. de Castro y F. J. Díaz (coords.), Universidad, sociedad y mercados globales (pp. 394-408). https://dialnet.unirioja.es/descarga/articulo/2752466.pdf

Schiffman, L. G., y Kanuk, L. L. (1991). Comportamiento del consumidor. Prentice-Hall Hispanoamericana.

Secretaria d'Universitats i Recerca. (2019). Universidades de Cataluña.

http://universitatsirecerca.gencat.cat/es/01_secretaria_duniversitats_i_recerca/universitats_i_re cerca_de_catalunya/universitats/universitats_de_catalunya/index.html

Segura-Mariño, A. G., Paniagua Rojano, F. J., y Fernández-Sande, M. (2020). Metodología para evaluar la comunicación universitaria en Facebook y Twitter. Prisma social, 28, 127-144. https://revistaprismasocial.es/article/view/3357/4084

Simón Onieva, J. E. (2014). El uso de las Redes Sociales en el ámbito de la comunicación universitaria andaluza. Revista internacional de relaciones públicas, 8, 139-160. https://doi.org/10.5783/RIRP8-2014-08-139-160

Simón Onieva, J. E. (2015). Gabinetes de comunicación universitarios y redes sociales [Tesis Doctoral, Universidad de Almería]. https://www.educacion.es/teseo/mostrarRef.do?ref=1202340

Trullàs, I. (2015). Proposta i avaluació empírica d'un model de màrqueting universitari centrat en el suport organitzatiu percebut i l'ocupabilitat percebuda per l'estudiantat [Tesis doctoral, Universitat Politècnica de Catalunya]. https://upcommons.upc.edu/bitstream/handle/2117/96290/TITC1de1.pdf

Varghese, N. V. (2008). Globalization of higher education and cross-border student mobility. Research Papers IIEP, 1-33.

http://citeseerx.ist.psu.edu/viewdoc/download?doi=10.1.1.536.4194\&rep=rep1\&type=pdf

Wang, V. (2011). Marketing Educational Programs through Technology and the Right Philosophies. International Journal of Technology and Educational Marketing, 1(1), 50-59. https://doi.org/10.4018/ijtem.2011010104

Wankel, L. A., y Wankel, C. (2011). Higher education administration with social media: including applications in student affairs, enrollment management, alumni relations, and career centers. Emerald.

Zarco, C., Del-Barrio-García, S., y Cordón, Ó. (2016). Propuesta de rankings de universidades españolas en redes sociales. El profesional de la información, 25(4), 684-698.

https://doi.org/10.3145/epi.2016.jul.18 